\title{
Forensic analysis of characteristic features of disguised writing in fixing authorship
}

\section{Reeta R Gupta}

Senior Scientific Officer (Document), Central Forensic Science Laboratory (CFSL), Central Bureau of Investigation (CBI), CGO Complex, Lodi Road, New Delhi, India

*Corresponding Author: Reeta R Gupta

Email: reetacbi@gmail.com

\begin{abstract}
Questioned Document Examiner deals with various types of criminal cased for fixing the authorship. Among them examination of disguised writings has thrown a serious challenge in solving the criminal cases in order to fix its authorship. Forensic analysis of handwriting characteristic features in disguised nature of writing content is not an easy task for questioned document experts to understand the detail characteristic features of writing content for fixing authorship. The present write-up is an attempt to unfold the journey of critical examination of disguised writings in order to reveal the authorship of written content. A large number of disguised writing has been examined for the purpose of detailed study of its characteristic writing features. The experimental evidence of disguised writing examination and analysis in identifying characteristic features related to disguised nature of writings content is discussed in detail in this research paper.
\end{abstract}

Keywords: Disguised writing, Forensic analysis of disguised writings, Document examination, etc.

\section{Introduction}

Questioned Document Examiner deals with various types of criminal cased for fixing the authorship. Among them, scientific examination and forensic analyses of disguised nature handwriting content in order to understand the distinctive features inherent in handwritten forgeries has thrown a serious challenge in solving the criminal cases in order to fix authorship. Writing characteristics that are individual to each writer and this individual characteristic deviates from the copybook norm which is easily possible to identify in normal writing content of any author handwritings written during normal course of business. However, in case of disguised handwriting, it is the willful modification of a person's natural writing for the purpose of concealing identity. The act of disguised is varied in nature and thus there is need to study the detailed characteristic features of disguised writings for significant individual characteristics of complex writing content. Before compiling the result the forensic document examiner has to deeply probe into the writing in order to find the minute and inconspicuous features, and other important writing characteristic in execution of stroke while making letter or in combination of letter/word accidently left by the writers. It is not possible to distort handwriting in such a manner as forger tries to conceal his/her identity completely, since handwriting is an output of neuromuscular co-ordination of brain with the hand of individual writer. Disguised writing may be viewed as a special form of forgery, since writer deliberately tries to alter the genuine writing for the purpose of hiding the personal identification. The complexity arises during the process of examination and identification of general as well as individual writing characteristic features, in analyzing the range and extent of natural variation in disguised writing. Thus, examination of handwriting contents which are disguised in nature is not an easy task for a forensic document expert. The objective of this paper is to explore the possibility of all important characteristic features available in a disguised writing content. The present write-up deals with the detailed/practical examination of various types of disguised writing encountered by the author during the course of their examination. The principle of handwriting science was applied for examination of disguised writing. On an average, in examination of disguised writing, a forensic document examiner always requires to do more hard work so as to collect wide variety of data available between questioned writing and standard writing. In examination of disguised writing one of the difficult issue is to accumulate all the handwriting features from the standard writing where the writer tries to change their own writing feature either by distorting stroke or changing in formation of letters etc. Osborn (1929) discusses the possible causes of error in forming an opinion of identification of writing if the forensic examiner observes differences which can be attributed to intended disguise. ${ }^{1}$ According to literature incorrect determination of disguise will occur when consistent and suitable characteristic feature leads to the differences between questioned and specimen or admittedly genuine/known writings which are attributed to the disguise when carefully not examined. ${ }^{2-5}$ Harison (1962) has stated that the change of capital from cursive to block letter is a popular device in disguised writing. ${ }^{5}$ According to Harrison corresponding characteristic features observed between questioned and known writings are being dismissed as being the two different writers when they are indicative of disguised by one writer. A principle and theory of forensic identification includes a thorough discussion of features of handwriting, such as pen scope and writing impulse, which are the basis of handwriting examination. ${ }^{6}$ According to Morris, the indications of disguised writing are mistaken for the sign of slowness or for the individual writer of lower skill. Morris explains the term graphic maturity that the degree of maturity of person's writing which is dependent upon age, physiology and experience. ${ }^{4}$ Koppenhaver (2002) explains that alteration of 
writing is closely associated with the alteration of speed. ${ }^{8}$ According to Hayes the modification of individual letter forms, it is more difficult than disguise to maintain, however the pictorial appearance can be altered. ${ }^{9-10}$ At the elemental level, during the process of comparison and examination sometimes it is also found that both questioned and standard writings, the disguised nature of writing content pertain some similar writing habit of stroke formation of letter which leads to individuality of writer.

\section{Characteristics Feature of Disguised Writing}

Disguised writing may be viewed as a special form of forgery, since writer deliberately tries to alter the genuine writing for the purpose of hiding the personal identification. The complexity arises during the process of examination and identification of general as well as individual writing characteristic features, in analyzing the range and extent of natural variation in disguised writing. In disguising the writing, the writer may believe that they have able to alter or change the entire characteristic feature of their writing. Numerous forensic experimental case works carried out by the author related with examination of signature and handwriting and explained herein. Most commonly, the disguisers wrote in a way that drastically changed the pictorial image of their writing. However the minute and conspicuous features were less likely to be disguised. Most common method of disguised was used by the disguiser: use of increased pen pressure throughout the writing content, alter letter forms, change punctuation and line spacing, wrote with notable tremor or erratic movements in an attempt to completely distort the writing or create an appearance of simulation. However during the examination of handwriting characteristic to characterizing it as disguised writing, it is must to justify it by overruling the fact of writing content examining the other reasons such as mental disorder, physical illness, intoxication and natural tremor occurring in writings. Apart from these factors, the expert should also focuses on examination of the fraudulent tremor related with disguised nature, opposite-hand writing, and attempt of tracing in handwriting etc. A large number of disguised writing has been examined to understand the characteristic writing features in such writings. In order to study the method applied by the disguiser in writing such writings and examination of detailed features of disguised writings reveals various changes in writing features such as: lack of uniformity of letter size, inconsistency in writing contents, unnatural pen pressure including much more other features. On the basis of forensic examination of such features in detailed, for an expert, it is possible to understand the disguised nature of writing content. Examination of various extensive disguised writings reveals that most of the disguised nature of writing and signatures is done by mean of changing the characteristic feature of the writing content such as by alteration in base line of signature, slant, beginning and ending stroke, degradation in line quality, the exclusive use of non-cursive upper class letter such as B,D,R,P, blunt start, blunt end, changed form of letter formation either by eliminating curve stroke or adding it from the staff e.g. B, b, D, d, R, r, P, p., Curve stroke eliminating from a letter's staff to form an arc or arches, e. g. m, h, $n$ etc. Changing in apex of letter where two ascending or descending strokes meet to form a joint often the highest part of letter such as A, M etc. It was also normally observed that in most of the disguise writing the making change in cross stroke i.e. a horizontal stroke that joins two strokes of a letter, such as $\mathrm{A}, \mathrm{H}$ and other letters in which cross stroke is horizontal that complete the letter, other letters such as J, E, F, T, t, f is more easier. Deviant writing i.e. false writing that deviate from naturally written writing in a manner to purposely produced by using combining all device of disguised act on which writing is made to be accepted as genuine.

During the examination of various handwriting contents related with disguised writings in routine case work, sometime the author encountered the difficulty in examination of finding significant handwriting characteristics features for fixing authorship. However due to consciousness during the act writing, by masking identity writer leave some point of their original hand characteristic feature which depend upon the skill of the writer gives also a very suitable identifying clue to fix the authorship. The relative relationship between a letter's vertical and horizontal measurement also distort. An attempt of to recorrection of strokes retouching or small delicate patching's very common in disguised writings. It is also observed that when individual disguises their own signature or sign fictitious signatures, the examination of such signature is tough task to handwriting examiner. Change in pictorial appearance of letter, deviation in writing stroke, degradation in line quality as well as inconsistency in the writing content including above mention other features are basis for the examination of disguised writing. With all effort of application of Principle of hand writing science and experience of the handwriting examination the forensic document examiner become able to definitely the author.

\section{Experimental Cases: Signature Examination:}

Genuine signature that are written by the signatory are deliberately and purposely changed to fulfill the mean of forgery or fraud i.e. disguised by forger in order to deny authorship at some later time to take benefit of doubt also. In case of signature forgery the disguiser tried employ the superfluous ornamentation as well as tries to make change in formation of stroke of letters with distortion of strokes. One of the experimental case consisting of questioned document marked Q-1 and Q-2, Specimen document marked S-1 to S-8 and Admitted document marked A-1 to A-8 is illustrated herewith related with the signature examination. Both the questioned and standard signatures were compares with each other in for their general and individual writing characteristic features. Examination revealed that questioned documents marked Q-1 and Q-2 are written freely and showing smooth line quality and natural variations. On the other hand the specimen signatures are disguised in nature. Admitted signatures are comparable with the questioned signatures. Detailed writing 
characteristic features including linear and angular measurement of writing strokes in execution of questioned signature marked Q-1 and Q-2 (Shown in Fig. 1) was compared with the Standard mark S-1 to S-8 (Shown in Fig. 2) and A-1 to A-A7 (Shown in Fig. 3) to understand the degree of execution of stroke in letters.

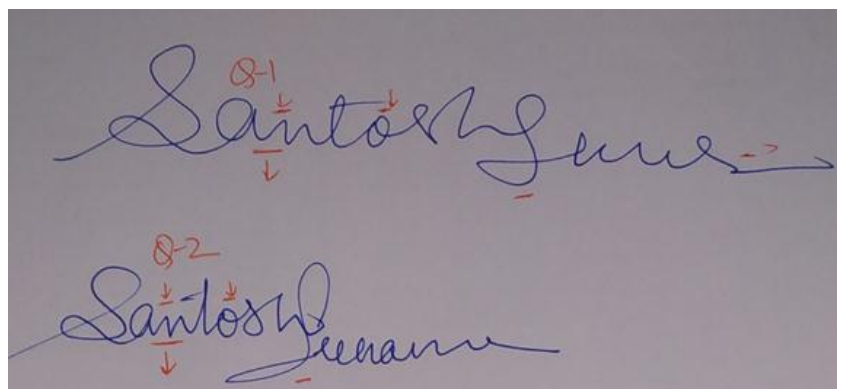

Fig. 1: Showing Questioned Signatures marked Q-1to Q2

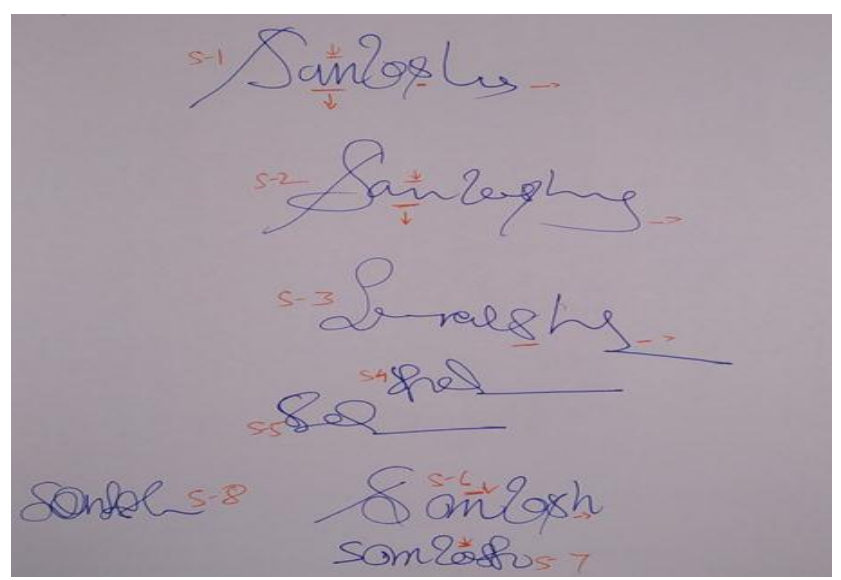

Fig. 2: Showing Specimen Signatures marked S-1to S-8

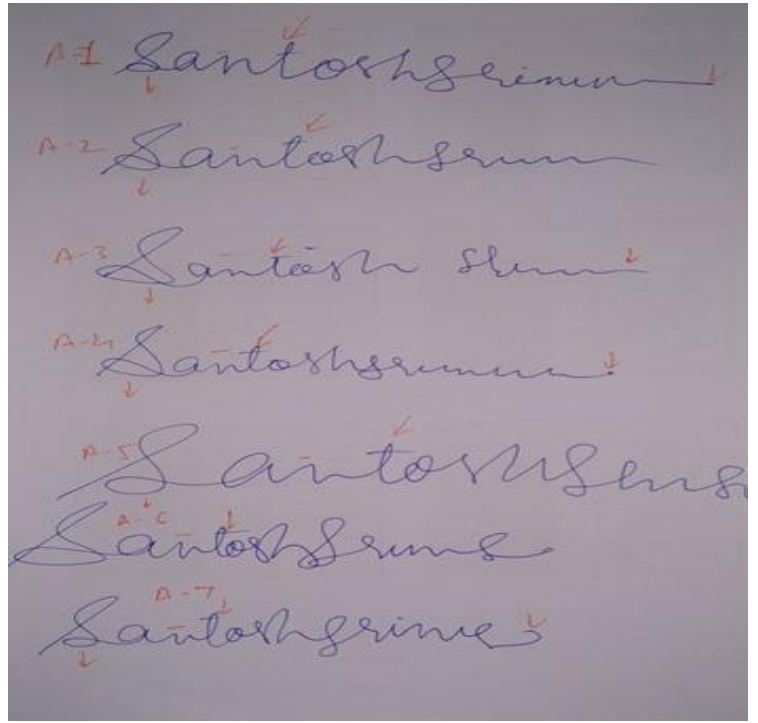

Fig. 3: Showing Admitted Signatures marked A-1to A-7
The authorship of the write was established on the basis of forensic analysis of general and individual characteristic features available in both sets of signatures. The linear measurement of both the sets of signatures questioned and standard were carried out with thorough scientific examination and comparison which includes linear dimensions of stroke in letters formation with horizontal, vertical and diagonal measurements of each stroke in formation of letter by calculating overall horizontal measurement, mid-zone height and relative spacing with relative height. The nature of execution of signatures marked Q-1 and Q-2 with nature of initial letter " $\mathrm{S}$ " and its relative relationship of letter " $a$ " with nature of connecting stroke as well as relative position as observed in questioned signature is similarly observed in admitted signatures marked A-1 to A-7. Similarity is observed in execution of combination of letter " $a$ " with letter " $n$ " both in questioned and standard signatures (specimen and admitted signatures). The writer tries to change the pictorial appearance of execution of letter " $t$ " in the specimen signatures. However, in admitted signatures this letter is similarly observed in admitted signatures. Furthermore, the execution of letters and combination of letters " $t$ " "o" "s" " $h$ " with relative shape, size and position is similarly observed in both questioned and admitted signatures, while in specimen signature writer tries to portray the letters. Rest of the letters signature is executed in highly simplified manner in between questioned and admitted signatures. Significant similarity is observed in termination strokes between both the sets of signatures. The details of significant characteristic features of both questioned and standard signatures are listed in Table 1. 
Table 1: Showing Comparison Chart of Significant Characteristic Features of Questioned Signatures and Standard Signatures

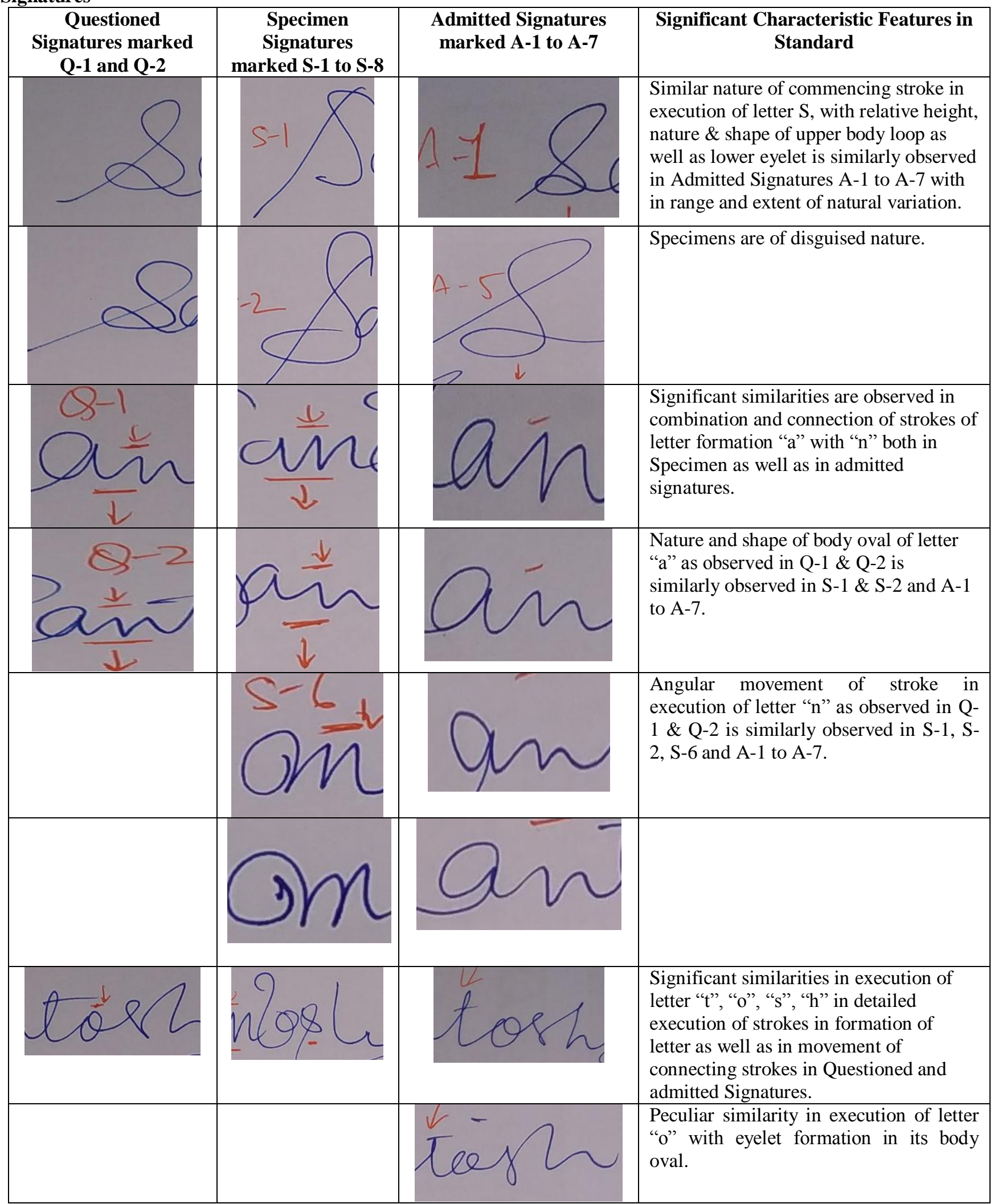




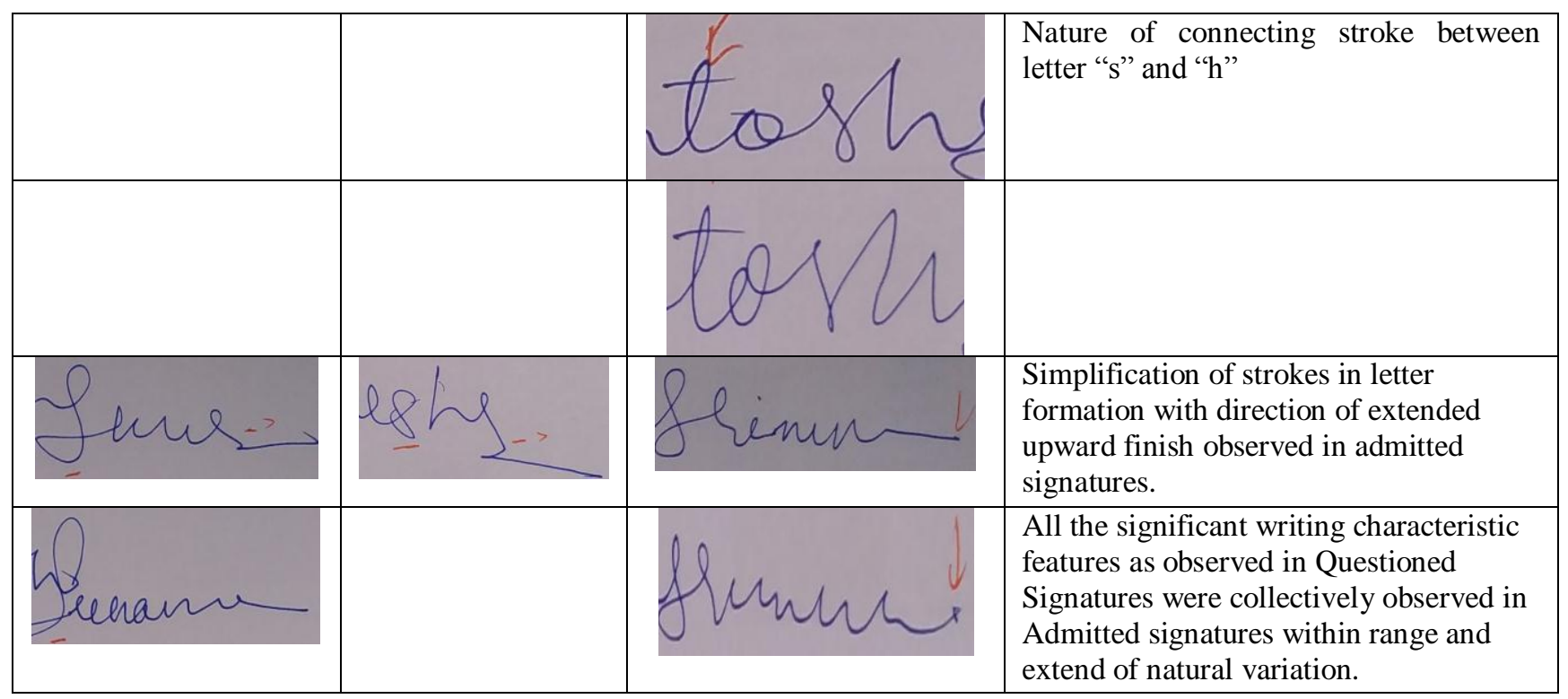

\section{Results}

Examination of disguised writings is really challenging task for the document examiner as expert is dealing with unusual and unnatural type of writings. A number of elements combine to form the pictorial appearance of writing stroke which includes the relation shape size proportion of letters, slant, strokes sequences that are used to construct the letters and connecting strokes that linked the letters in execution of signatures or words. Many of the cases examined by the authors of suspected writing it was apparent during the course of examination that the person doing the act of disguised try to add some unknown characteristics and hide their own characteristics, hence produce distorted version of writing of the same writing content. Henceforth due to lack of knowledge he/she create a letter, words, together with a lack of physical and mental disturbance which impact on disguised writing content. Because of consciousness of mind the consistence of written content also disturbed. Thus inconsistency in the writing content gives important clue to suspect the examiner for disguise nature of writing. The deliberately misspelling of words is also notice in the disguised nature of writing. Unnatural variation in writing size also randomly occurred in disguised writing during the act of alteration the normal form of letter change and overall size increased. Unusual enlargement of size is also one of the important factors in disguised writing content. Most of the disguise cases the attempts made by writers to mislead fact by using any of methods such as retouching and overwriting, change in movement, slant alignment, misspelling of words, change of letter formation, change of writing instrument, blunt finish, change angle of curved stroke, increase in letter size, hand printing, connecting stroke modification including initial and terminal stroke modification, change of small letter into capital letter, change of arrangement of text character, omission or addition of letter in word, and much more effort, the forger make is very complex in nature, so identification of author of such signature and writing become more difficult.

The illustrated experimental case examination reveals that the writer some time used specific tactic by changing the pictorial appearance of the letter in executing specimen signature in order to mislead the fact. In this experimental cases discussed herewith, an attempt of disguised method is recognizing its characteristic features in disguised nature of signatures; it was found present in specimen signatures. In this disguised nature of specimen signatures the initial strokes of first letter "S" of signature are more susceptible to alter/change by the writer. Major changes and modification was focused to the strokes of some specific letters such as " $t$ " and somehow in execution of letter "o". Results disclosed by experimental case there is inconsistency of strokes in letter formation in specimen signatures. The experimental evidence of disguised signatures examination and analysis suggested that while in identifying characteristic features in any disguise signature/writings, pictorial dissimilarities can be ignored sometimes, beside that minute \& inconspicuous characteristic features of similarities should be carefully taken into consideration for conclusion of the opinion in disguised nature of writing. Examination of the experimental case illustrated above, the scientific principles of handwriting science along with experience of field was applied. The result shows that all the pictorial dissimilarities occurring in a specimen signature is due to intention of writer to hide their own individual writing characteristic features. On account of the aforementioned scientific evidence, it is concluded that the authorship of questioned signatures and standard signatures are one and the same person. Significant individual writing characteristic features of available between both questioned signatures and admitted signatures was the scientific basis for fixing authorship. 


\section{Conclusion}

The illustrated experimental case, mention above is only example to understand the significant writing characteristic features in disguised nature of examination. From the experience of the cases examined by the authors in many cases, it is suggested that admitted signatures and writing of contemporary period should asked to understand the writing habit of writer. If sufficient and suitable number of standards has been available for the purpose of its comparison with questioned documents, the chance of repeat and repeat availability of identifying characteristic features increased certainly it facilitate the examiner to come on some concrete conclusion in order to fix authorship. Success in concealing or camouflaging one's identity in writing characteristic features, and therefore the examination through the process of forensic handwriting comparison and applying the principle of handwriting science, depends not only upon the extent one has modified his/her handwriting, but also, the nature of the questioned writing, the available material for comparison, and the experience, skill and competency of the forensic document examiner. Characteristic difference between the questioned and standard regarding detection of the author identification in disguised writing sometime need more focused examination and hence more standard writings should be further taken in scientific comparison with questioned document so that all significant characteristic features should be possible to account far. Reason to understand the differences or similarities in two sets of writing is must because some factor affects the writing characteristics such as ill health of writer, influence of alcohol, external factor i.e. writing surface etc. Thus while considering the disguised writing, these all other factor should also be taken in notice to rectify the chance of any possible error. On the basis of above discussion it is concluded that most attempts to disguise writing the disguisers are neither consistent nor successful.
Conflict of Interest: None.

\section{References}

1. Questioned Document. Albert S. Osborn. Nelson-Hall Co., Chicago, III.,1929

2. Wilson R Harrison (1966), page 349, Suspected Document.

3. Devid Ellen1997, p-51, The Scientific Examination of Documents: Methods and Techniques, second Edition, CRC Press LLC.

4. Morris RN (2000) Forensic Handwriting Identification: Fundamental Concepts and Principles. Academic Press, USA

5. Wilson R Harrison (1997), Suspected Document: Their Scientific Examination, Second Indian Reprint, Universal Law Publishing Co. Pvt. Ltd.

6. Ordway Hilton (1993), Scientific Examination of Questioned Document, Revised Edition, CRC Press.

7. Jay Siegal, geoffery Knupfer, Pekka Saukko (2000), Encyclopedia of forensic sciences, three volume set Elsevier.

8. Katherine Koppenhaver 2002 Attorney's Guide to document examination

9. Hays 2006 p-165 Forensic Handwriting Examination -A Definitive Guide. ReedWrite press, Honolulu, USA.

10. Ellen D (2006) scientific Examination of Documents- Methods and Techniques, (3rd edn), CRC Press- Taylor \& Francis group, USA.

11. J. V. Convey, (1978), Evidential Documents, Charles C Thomas Publisher Ltd.

12. Dowson, G A (1985) Brain function and writing with the Unaccustomed left Hand, J Forensic Sci 30;167-171.

13. Disguised Handwriting: A statistical Survey of How handwriting is most frequently disguised. Edwin F. Alford, Jr.

14. Disguised writing and its examination: National Forensic Science Conference LU India.(2017) Reeta R Gupta, N Ravi.

15. Forensic Examination of Disguised writings: International conference Forensic research and Technology, Osaka, Japan 2018. Reeta R Gupta.

How to cite this article: Gupta RR, Forensic analysis of characteristic features of disguised writing in fixing authorship. Int J Forensic Med Toxicol Sci 2019;4(1):22-27 